\title{
ADULT-ONSET STILL'S DISEASE AND FRANTZ'S TUMOR AN UNPRECEDENTED ASSOCIATION
}

Bruna Martins Liberali1,", Noemy Matos Hirokawa ${ }^{1}$, Vardelei Segatelli ${ }^{2}$, Ludmilla de Oliveira Muniz Koch², Eduardo Figueiredo Benedetti², Roberto Ferreira Meirelles Junior ${ }^{2}$, Luiz Felipe Adsuara de Sousa ${ }^{1,2}$

1. Universidade Anhembi Morumbi, São Paulo (SP), Brazil. 2. Hospital Israelita Albert Einstein, São Paulo (SP), Brazil.

*Corresponding author: brunaliberali@gmail.com

\section{BACKGROUND}

Adult-onset Still's disease (AOSD) is a rare systemic inflammation, characterized by fever, rash, arthritis, lymphadenopathy, hepatosplenomegaly, myalgia, odynophagia and leukocytosis. There are no specific laboratory markers, but hyperferritinemia and elevated inflammation markers can be found. Frantz's tumor of the pancreas is a rare neoplasia that affects young females, mainly on body-tail zone of pancreas, with low malignant potential and favorable prognosis after surgical resection.

A case of a male patient diagnosed with AOSD and Frantz's sumor is reported. This is the first case reported with this association. This work aimed to identify the possibility of AOSD as paraneoplastic syndrome of Frantz's tumor or if both of them expressed apart in the same patient.

\section{CASE REPORT}

A 33-year-old men, without personal antecedents, searched the service on 2020 February with odynophagia 3 weeks ago with erythematous rash on hands and upper limbs. Sought medical service and was prescribed glucocorticoid, evolving with partial improvement. Began to experience dry cough, fever, and worsening of the rash, then sought infectious disease service, when performed a negative oropharynx culture. During this period, he used also antihistamines with partial improvement. He started to have myalgia, migratory knee pain and elbow pain, left proximal interphalangeal joints arthritis.

Rheumatology team ordered laboratory tests. Among the altered exams, there were serum ferritin ( $>16.500 \mathrm{ng} / \mathrm{mL})$, erythrocyte sedimentation rate $(68 \mathrm{~mm})$, C-reactive protein $(59.9 \mathrm{mg} / \mathrm{dL})$ and hepatosplenomegaly. Research was negative for autoimmune and infectious diseases.

With the diagnostic hypothesis of AOSD, the dose of prednisone ( $60 \mathrm{mg} / \mathrm{day}$ ) were increased with complete improvement. However, at prednisone weaning for $40 \mathrm{mg} / \mathrm{day}$, the patient had a significant change in the transaminase levels ( $283 \mathrm{and} 806 \mathrm{U} / \mathrm{L}$ ). The patient was hospitalized to start treatment with $500 \mathrm{mg} /$ day of methylprednisolone for 3 days, and to exclude other causes of hepatitis.

During this hospitalization, injury to the pancreas was evidenced with $2 \mathrm{~cm}$ at abdomen magnetic resonance. The tumor was biopsied and evidenced as Frantz's tumor. After resection, occurred relapse of hepatitis after decreasing prednisone to $45 \mathrm{mg} / \mathrm{day}$.

\section{CONCLUSION}

The differentiation between concomitance of diagnoses and paraneoplastic syndrome is not simple. However, the therapeutic response to the specific treatment to the tumor can help to differentiate it. In the paraneoplastic syndrome, after neoplasm treatment, there is usually remission or considerable improvement of the syndrome. In this case, AOSD apparently remained after tumor resection. There are many reports in literature of AOSD as paraneoplastic syndrome, but this case is the first report of AOSD associated with Frantz's tumor. 